\title{
ASPECTOS ALIMENTARES E DE CRIAÇÃO DE BRADYSIA HYGIDA SAUAIA \& ALVES (DIPTERA, SCIARIDAE) EM LABORATÓRIO
}

\author{
Iara S. Joachim Bravo 1 \\ Maurílio A. R. Alves 1 \\ Fernando S. Zucoloto 1
}

Luiz A. M. Andrade 2

\begin{abstract}
ASPECTS ON ALIMENTATION AND REARING OF BRADYSIA HYGIDA SAUAIA \& ALVES (DIPTERA, SCIARIDAE) IN LABORATORY. In nature, the Sciaridae live in moist and shady places, where exists vegetable material in decomposition. It is very dificult to determine the proper Sciaridae's alimentary habit and then, it is dificult, also, to rear Sciaridae species in laboratory. To improvement the Bradysia hygida rearing, the objectives of this research were to analyse the nutritive value of mucuna bean foliage for the larves, to verify the role of the ground (as larvae substrate and in diet composition) and also to verify the possibility of ground substituition by another kind of substrate. Two kinds of sand were employed in the place of the ground and three diets, free of ground, were prepared. The parameters analysed were: duration of the life cicle, porcentage of emergence, egg production by female and adult size. The results showed that the mucuna bean foliage have a good nutritive value for B. hygida rearing; the ground, as substrate, can be substituted by any of the sands and the ground, in the diet, is also dispensable. The alimentary habits of Sciaridae is discussed.

KEY WORDS. Diptera, Sciaridae, Bradysia hygida
\end{abstract}

A família Sciaridae é encontrada geralmente em lugares úmidos e sombrios. Apresenta ampla distribuição geográfica e sua presença na natureza está ligada principalmente à existência de material vegetal em decomposição, decorrendo daí, sua importância na ecologia do solo (STEFFAN, 1974; KENNEDY, 1974; BINNS, 1981; DELEPORTE \& ROULAND, 1991). Algumas espécies desta família são conhecidas como pragas de várias plantas cultivadas e de algumas espécies de cogumelos comerciais (WILKINSON \& DAUGHERTY, 1970; GRHAN \& MCNEIL, 1972; DENNIS, 1978; RUTHERFORD et al., 1985; CANTELO, 1988; KEIL \& OTHMAN, 1988), tendo isso importância econômica.

Pouco se conhece sobre os hábitos alimentares naturais e sobre os métodos de criação dos Sciaridae em laboratório. Alguns procedimentos já

1) Departamento de Biologia, Universidade Estadual de São Paulo, 14040-030 Ribeirão Preto, São Paulo, Brasil.

2) Departamento de Morfologia, Universidade de São Paulo, São Paulo, Brasil. 
foram descritos (LARA et al., 1965; MORGANTE et al., 1970; STEFFAN, 1974), porém, todos eles implicam em dietas de difícil controle, que no geral acarretam problemas na manutenção contínua da criação desses insetos.

O Sciaridae Bradysia hygida (SAUAIA \& ALVES, 1968), tem sido criado no laboratório desde 1965, com a finalidade de fornecer material biológico para o estudo dos pufes de DNA. Suas larvas têm sido alimentadas basicamente com rama de batata doce (Ipomoea batatas) seca, moída e fermentada colocada sobre terra úmida dentro de caixas plásticas. A rama de batata doce é obtida em chácaras próximas à cidade de Ribeirão Preto e como produto comercial, é cultivada com a utilização de agrotóxicos. Durante todos esses anos, a criação de $B$. hygida sofreu vários problemas de manutenção, com baixa porcentagem de eclosão de larvas, aumento da duração do ciclo de vida dos insetos, rejeição do alimento por parte das larvas e alta mortalidade destas. Em certas ocasiões esses problemas chegaram a colocar em risco a manutenção da criação. Tais dificuldades foram atribuídas à inconstância da qualidade da rama de batata doce e à possível contaminação da terra usada como substrato para criação das larvas.

Com a finalidade de conseguir dados que pudessem contribuir para a melhoria das condições de criação da $B$. hygida foi planejado o presente trabalho. Ele foi dividido em duas partes. Na primeira, o objetivo foi testar o valor nutritivo de uma rama diferente daquela da batata doce - a do feijão mucuna (Mucuna pruriens D.C.). Ela foi escolhida por ser uma planta selvagem, tendo condições de ser cultivada em locais próximos do laboratório e sem defensivos agrícolas. Além disso, em testes preliminares, misturada com a rama de batata doce e adicionada com levedo de cerveja, maizena e farinha de aveia, mostrou ser aceita pelas larvas, permitindo o fechamento do ciclo de vida do inseto.

Na segunda fase, o objetivo foi avaliar a necessidade da terra utilizada como substrato e como componente das dietas. A terra sempre foi misturada à rama de batata com o objetivo de melhorar suas qualidades alimentares. Como substrato, ela tem sido mantida porque as larvas, no quarto estádio, deixam a comida, ingerem-na e constroem o pupário, usando para isto, terra e secreção salivar.

Assim, este trabalho pretende verificar: a possibilidade de substituição da rama de batata doce por outra rama; a possibilidade de substituir a terra por outros substratos; a importância da terra como fonte de nutrientes para as larvas e para a qualidade das ramas e a possibilidade de padronização de dietas sem terra, o que lhes daria condições de controle mais eficientes.

\section{MATERIAL E MÉTODOS}

\section{Parte I}

Foram formados três grupos, cada um com 100 larvas recém-eclodidas. A cada grupo foi oferecida uma de três dietas:

dieta "A" - rama de batata doce, seca moída e fermentada. 
dieta "B" - rama de feijão mucuna, seca moída e fermentada.

dieta "C" - mistura das duas ramas adicionadas de levedo de cerveja, farinha de aveia e maizena - dieta controle.

As três dietas foram preparadas da seguinte maneira: em recipiente de polietileno $(22 \times 22 \times 10 \mathrm{~cm})$ colocava-se rama (de batata doce ou mucuna) seca e triturada $(300 \mathrm{~g})$ e terra peneirada $(100 \mathrm{~g})$; à mistura adicionava-se água de torneira em quantidade suficiente para umidecer. À dieta controle (preparada como as anteriores) acrescentava-se farinha de aveia $(5 \mathrm{~g})$, levedo de cerveja $(5 \mathrm{~g})$, e maizena $(5 \mathrm{~g})$. As caixas eram cobertas com tecido de algodão e permaneciam em processo de "fermentação" por cinco dias, em sala a $20^{\circ} \mathrm{C}$.

A montagem das caixas de criação e o tratamento das larvas obedecia o seguinte: as dietas eram colocadas sobre terra úmida em caixas de acrílico $(11 \times 11 \times 3,4 \mathrm{~cm})$, onde também eram colocadas as larvas recém-eclodidas. Durante todo o desenvolvimento cuidava-se para que não faltasse alimento, adicionando-o se necessário. Dentro da caixa a umidade era superior a $70 \%$, a temperatura da sala de criação era de $20 \pm 1^{\circ} \mathrm{C}$ e o fotoperíodo de 12 horas claro/escuro.

Os parâmetros utilizados para a avaliação do valor nutritivo das dietas foram os seguintes: duração do ciclo de vida, porcentagem de emergência, número de óvulos por fêmea e tamanho do adulto (estimado pelo comprimento da asa). Para a análise do número de óvulos e tamanho do adulto, 20 fêmeas recém-emergidas, de cada dieta, eram fixadas em Fixador Dietrisch (álcool etílico $95 \%$ - 55ml; formol - 5ml; ácido acético glacial - $5 \mathrm{ml}$ e água destilada $35 \mathrm{ml}$ ) por 24 horas.

Os experimentos foram feitos por 10 gerações sucessivas e para cada grupo fez-se três réplicas.

Para análise dos resultados os testes estatísticos utilizados foram Freedman à 5\% de significância, (HOLLANDER \& WOLFE, 1973) para comparação das dietas em cada geração e ManWhitney à 5\% de significância (SIEGEL, 1975), para comparação das dietas entre a primeira e a décima gerações.

\section{Parte II}

Nesta parte testou-se três dietas preparadas sem terra e dois tipos de areia, como substratos alternativos à terra. As dietas foram as seguintes: dieta mucuna - rama de feijão mucuna seca e moída; dieta batata - rama de batata doce seca e moída; dieta mistura - ramas de batata doce e feijão mucuna secas e moídas. Todas as dietas eram lavadas com água corrente, exaustivamente, (para eliminação da terra) e autoclavadas $\left(120^{\circ} \mathrm{C} / 20 \mathrm{~min}\right.$.). Posteriormente, eram guardadas em caixas de polietileno, onde "fermentavam" por cinco dias antes de serem oferecidas às larvas. Como controle foi usada a mesma dieta controle da parte I.

Como substrato foram utilizadas as seguintes areias: areia $\mathbf{A}-0,45 \mathrm{~mm}$ de diâmetro, obtida no serrado de Santa Rita do Passa Quatro, São Paulo e antes 
de ser utilizada era lavada em água corrente por 24 horas; areia B $-0,074 \mathrm{~mm}$ de diâmetro, é uma areia de quartzo da marca Ki-Kion. Como controle foi usada a terra roxa das proximidades do laboratório.

Foram montados 12 grupos de 100 larvas recém-eclodidas (cada grupo composto por três caixas de criação - réplicas), combinando-se as quatro dietas com os três substratos. As caixas de criação, montadas, receberam tratamento semelhante ao descrito na parte I. Também, para comparação das dietas e dos substratos, analisou-se os mesmos parâmetros já mencionados na parte I. Ainda, à semelhança da parte $\mathrm{I}$, os experimentos foram realizados por 10 gerações sucessivas. A análise estatística foi feita da seguinte maneira: para os parâmetros ciclo de vida e porcentagem de emergência foram obtidos dados das gerações um, dois, nove e 10. Os três substratos e as quatro dietas tiveram análise independente. A comparação dos substratos foi feita com dados das gerações um e 10, enquanto que, a comparação das dietas com os dados das gerações dois e nove. A montagem dos experimentos não permitiu a análise de substratos e dietas em uma mesma geração. Para a análise dos parâmetros números de óvulos e tamanho do adulto utilizou-se somente os dados das gerações um e 10, sendo que, das 20 moscas fixadas, 10 entraram na comparação dos substratos e as outras 10 na comparação das dietas. Os testes estatísticos foram: Mann-Whitney (SIEGEL, 1975) à 5\% de significância, para verificar a existência de diferenças entre as gerações um e dois; nove e 10, a fim de que as análises entre os diferentes substratos e as diferentes dietas pudessem ser realizadas empregando dados de gerações diferentes, mas equivalentes; Kruskal Wallis (SIEGEL, 1975) e Kruskal Wallis para comparação múltipla (HOLLANDER \& WOLFE, 1973), ambos à 5\% de significância, para a comparação entre os substratos e, também, entre as dietas. Tais testes foram escolhidos pelo fato dos dados não apresentarem distribuíção normal.

\section{RESULTADOS E DISCUSSÃO}

\section{Parte I}

Os resultados desta parte estão sumarizados na tabela I. Pelos dados obtidos e pela análise estatística podemos concluir que as três dietas são igualmente adequadas para os parâmetros: porcentagem de emergência, número de óvulos e tamanho do adulto.

Para o parâmetro ciclo de vida houve diferença entre a dieta controle e a dieta de rama de batata doce, sendo que, o ciclo de vida com esta última é maior. Este resultado sugere que a dieta "A" (rama de batata doce) é menos adequada que as demais, isto porque, a duração do ciclo de vida depende, entre outros fatores, da qualidade da alimentação e, quanto melhor ela for, menor a duração do ciclo, obviamente, dentro dos limites da espécie (ROCK, 1972).

A dieta "B" (rama de feijão mucuna) considerando-se ainda o parâmetro duração do ciclo de vida, não mostrou diferença em relação a dieta controle (ao contrário da batata), o que sugere que a rama de feijão mucuna é mais adequada 
que a de batata. Isto é vantajoso porque a plantação do feijão mucuna não requer os cuidados de uma cultura agrícola, o que ocorre com a batata doce. Faz-se necessário ponderar, no entanto, que a rama do feijão mucuna, à semelhança da de batata doce, é uma dieta de controle difícil, pois, até o presente não se conhece os microrganismos envolvidos nos seus processos de fermentação. Porém, tanto a rama do feijão mucuna como a de batata doce, mantém de modo contínuo, a criação de $B$. hygida, o que não ocorreu com as dietas artificiais (FRANCISCO \& ZUCOLOTO, 1985; TRAMONTE \& ZUCOLOTO, 1988) testadas até agora para criação desta espécie.

Tabela I. Resultados obtidos no desenvolvimento de Bradysia hygida alimentada com as dietas "A" (rama de batata doce), "B" (rama de feijão mucuna) e "C" (controle). Foram analisados os seguintes parâmetros: duração do ciclo de vida, porcentagem de emergência, número de óvulos por fêmea e comprimento da asa. Os números representam a média e desvios padrões dos dados de 10 gerações sucessivas.

\begin{tabular}{ccccc}
\hline Dieta & $\begin{array}{c}\text { Ciclo de vida } \\
(\text { dias })\end{array}$ & $\begin{array}{c}\text { Emergência } \\
(\%)\end{array}$ & $\begin{array}{c}\text { Número de } \\
\text { óvulos/fêmea }\end{array}$ & $\begin{array}{c}\text { Comprimento } \\
\text { da asa }(\mathrm{mm})\end{array}$ \\
\hline A & $29.00 \pm 2.34^{*}$ & 66,51 & $194,02 \pm 3,72$ & $2.75 \pm 0.06$ \\
B & $27.90 \pm 1,91$ & 68,45 & $191,01 \pm 6,98$ & $2,76 \pm 0,03$ \\
C & $26.40 \pm 0.96^{*}$ & 70.31 & $183,04 \pm 6,33$ & $2,74 \pm 0.04$ \\
\hline
\end{tabular}

* Valores estatisticamente diferentes entre si

\section{Parte II}

Os resultados da parte II estão sumarizados nas tabelas II e III.

Com relação à comparação dos três substratos, a análise estatística mostrou que: para o parâmetro duração do ciclo de vida houve diferença entre os três substratos somente em dois casos - na dieta mucuna $\mathrm{F}_{1}$ e na dieta batata $\mathrm{F}_{10}$ - no primeiro, as larvas criadas na areia $\mathbf{A}$ tiveram ciclo mais longo que as da areia B; no segundo, as larvas criadas com areia $\mathbf{B}$ tiveram ciclo mais longo que as da terra.

Para o parâmetro porcentagem de emergência não houve diferenças significativas entre os três substratos.

No parâmetro número de óvulos por fêmea, houve diferenças em quatro ocasiões: na dieta mucuna $F_{1}$ as moscas da areia $\mathbf{B}$ apresentaram mais óvulos que as dos demais substratos. Na dieta batata $F_{1}$, as moscas da areia $\mathbf{A}$ apresentaram menor quantidade de óvulos que as dos demais substratos. E nas dietas controle $F_{10}$ e batata $F_{10}$ as moscas criadas em terra apresentaram maior número de óvulos que as dos demais.

Para o parâmetro tamanho do adulto houve diferenças significativas em sete casos ( $\mathrm{Tab}$. II), sendo que, nas dietas controle $\mathrm{F}_{1}$ e $\mathrm{F}_{10}$ as moscas da areia B foram menores que as dos outros substratos. Contrariamente, nas dietas mucuna $F_{1}$ e $F_{10}$ as moscas da areia $\mathbf{B}$ foram maiores que as demais. Na dieta 
Tabela II. Duração do ciclo de vida, porcentagem de emergência, número de óvulos por fêmea e comprimento da asa de Bradysia hygida criada em três substratos diferentes com cada uma das quatro dietas. Os números representam as médias e desvios padrões de três repetições contendo 100 larvas cada uma, nas gerações um e 10.

\begin{tabular}{|c|c|c|c|c|c|c|c|c|c|}
\hline \multirow{2}{*}{ Dieta } & \multirow{2}{*}{ Substrato } & \multicolumn{2}{|c|}{ Ciclo de vida (dias) } & \multicolumn{2}{|c|}{ Emergência ( $\%$ ) } & \multicolumn{2}{|c|}{ Número de óvulos/fêmea } & \multicolumn{2}{|c|}{ Comprimento da asa (mm) } \\
\hline & & $\mathbf{F}_{1}$ & $F_{10}$ & $F_{1}$ & F 10 & $F_{1}$ & F10 & $\mathrm{F}$ & F10 \\
\hline \multirow{3}{*}{ Controle } & Terra & $33,00 \pm 0,00 \mathrm{a}$ & $34,67 \pm 0,57 \mathrm{a}$ & $87.33 \mathrm{a}$ & $68,33 \mathrm{a}$ & $197,80 \pm 16,84 a$ & $192,60+9.42 a$ & $2.80 \div 0,02 \mathrm{a}$ & $2,74 \pm 0,08 a$ \\
\hline & Areia A & $33,00+0,00 \mathrm{a}$ & $31,00+0,00 \mathrm{a}$ & $81,33 \mathrm{a}$ & $76,33 \mathrm{a}$ & $194,70+8,35 \mathrm{a}$ & $166.10 \times 17,80 \mathrm{~b}$ & $2,79+0,02 \mathrm{a}$ & $2.71+0,04 \mathrm{a}$ \\
\hline & Areia B & $33,00+0,00 \mathrm{a}$ & $31,00 \div 0,00 \mathrm{a}$ & $80,33 \mathrm{a}$ & $77,33 \mathrm{a}$ & $186,20+22,20 \mathrm{a}$ & $159,30+16,57 \mathrm{~b}$ & $2.75+0.02 \mathrm{~b}$ & $2,63 \pm 0,05 b$ \\
\hline \multirow{3}{*}{ Mistura } & Terra & $32,00+0,00 \mathrm{a}$ & $34,00+0,00 \mathrm{a}$ & $89,33 \mathrm{a}$ & $79,67 a$ & $190,70+22,20 \mathrm{a}$ & $206,60+13,45 \mathrm{a}$ & $2.80 \pm 0,05 \mathrm{a}$ & $2,83+0,06 a$ \\
\hline & Areia A & $32,00+0.00 \mathrm{a}$ & $32,33 \pm 1,15 a$ & $82.33 \mathrm{a}$ & $83.67 \mathrm{a}$ & $190,60 \pm 5.02 \mathrm{a}$ & $198.10+15.37 \mathrm{a}$ & $2.79+0.05 \mathrm{ah}$ & $2.78+0,04 a$ \\
\hline & Areia B & $32,00+0,00 \mathrm{a}$ & $33,00+0,00 \mathrm{a}$ & $83,00 \mathrm{a}$ & $92,00 \mathrm{a}$ & $195,00+12,75 a$ & $214,30+12,41 \mathrm{a}$ & $2,77+0,05 b$ & $2,83+0,04 a$ \\
\hline \multirow{3}{*}{ Mucuna } & Terra & $34,67 \pm 0,57 \mathrm{ab}$ & $34,00+0,00 \mathrm{a}$ & $70.33 \mathrm{a}$ & $71.50 \mathrm{a}$ & $115,00+10,42 \mathrm{a}$ & $157,70+40,40 \mathrm{a}$ & $2.45 \pm 0.05 \mathrm{a}$ & $2.65+0,19 \mathrm{ab}$ \\
\hline & Areia A & $36,33+0,57 \mathrm{a}$ & $35,00+0,00 \mathrm{a}$ & $75,66 a$ & $63,00 \mathrm{a}$ & $104,70+11,83 \mathrm{a}$ & $150,50+39,42 \mathrm{a}$ & $2,39+0,05 a$ & $2,65+0,17 \mathrm{a}$ \\
\hline & Areia B & $30.00+0.00 \mathrm{~b}$ & $34,00+0,00 \mathrm{a}$ & $74.00 \mathrm{a}$ & $65,50 \mathrm{a}$ & $176.70+20.42 \mathrm{~b}$ & $191.90+32.83 \mathrm{a}$ & $2.66+0.11 \mathrm{~b}$ & $2.8 \mathrm{t}+0,09 \mathrm{~h}$ \\
\hline \multirow{3}{*}{ Batata } & Terra & $38,33 \pm 1,15 \mathrm{a}$ & $35,00+0,00 \mathrm{a}$ & $58,00 \mathrm{a}$ & $72.56 \mathrm{a}$ & $187.25 \pm 11.95 \mathrm{a}$ & $198.15 \pm 10,60 \mathrm{a}$ & $2,69+0.05 \mathrm{a}$ & $2,84+0.05 \mathrm{a}$ \\
\hline & Areia A & $38,33 \pm 0,57 \mathrm{a}$ & $36,00-0,00 \mathrm{ab}$ & $62,66 \mathrm{a}$ & $64.55 \mathrm{a}$ & $120,70+9,23 b$ & $179.50+16.77 \mathrm{~b}$ & $2,37+0,04 \mathrm{~b}$ & $2,71+0.05 b$ \\
\hline & Areia B & $37.67 \pm 0.57 a$ & $37,00+0,00 \mathrm{~h}$ & $56.67 \mathrm{a}$ & $65.67 \mathrm{a}$ & $159.60 \cdot 17.90 \mathrm{a}$ & $169.10 \cdot 12.81 \mathrm{~h}$ & $2.75 \cdot 0.05 \mathrm{a}$ & $2.75 \cdot 0.05 \mathrm{~h}$ \\
\hline
\end{tabular}

* Dentro de cada dieta, médias seguidas da mesma letra, em coluna, não diferem entre si (Kruskal Wallis, comparação múltipla à $5 \%$ ).

batata $F_{1}$ as moscas da areia $\mathbf{A}$ foram menores que as outras e nas dietas mistura $F_{1}$ e batata $F_{10}$, as moscas da terra foram maiores que as da areia $\mathbf{B}$, na primeira, e que as dos demais substratos, na segunda.

Em relação aos três substratos, areia $\mathbf{A}$, areia $\mathbf{B}$ e terra (controle), pode-se notar que as diferenças encontradas foram dispersivas. Apesar de ter havido diferenças entre eles, estas não foram constantes a ponto de indicar a superioridade de um substrato sobre o outro, em nenhum dos parâmetros analisados. Pode-se concluir, portanto, que todos os substratos tiveram o mesmo desempenho para o desenvolvimento das larvas.

Como os substratos são os locais de construção dos pupários, esperava-se que possíveis diferenças entre os três substratos analisados pudessem se manifestar na porcentagem de emergência, como ocorre com outros insetos. Em Ceratitis capitata e Dacus oleae a diferença de umidade e textura dos substratos afetaram as suas porcentagens de emergência (NEUENSCHWANDES et al., 1981; AZEVEDO \& PARRA, 1989). Também substratos muito compactos aumentaram a mortalidade de pupas de Heliothis zea e Diabrotica undecimpunctata howardi (LUMMUS et al., 1983; ROACH \& CAMPBELL, 1983). Isto porém, não ocorreu com os resultados deste trabalho, indicando que a porcentagem de emergência de $B$. hygida não é afetada pelo tipo de substrato. Estes resultados demonstraram que a terra pode ser substituída por qualquer uma das areias, que são substratos mais inertes. Por não ser inerte, a terra é um fator 
limitante para o bom desempenho de uma dieta artificial. $\mathrm{O}$ fato dela poder ser substituída abre possibilidades para a realização de experimentos com dietas artificiais, semelhantes aos trabalhos de FRANCISCO \& ZUCOLOTO (1985) e TRAMONTE \& ZUCOLOTO (1988), que proporcionam condições mais estáveis para as criações de insetos.

Com relação as dietas (Tab. III), a análise estatística mostrou que: para o parâmetro ciclo de vida houve diferenças significativas em seis casos, sendo que, em quatro deles as moscas criadas na dieta controle tiveram ciclo de vida menor que as da batata doce, e nos outros dois, as moscas criadas na dieta mistura e na dieta mucuna, respectivamente, tiveram duração do ciclo de vida menor que as da batata.

Tabela III. Duração do ciclo de vida, porcentagem de emergência, número de óvulos por fêmea e comprimento da asa de Bradysia hygida criada em quatro dietas diferentes com cada um dos três substratos. Os números representam as médias e desvios padrões de três repetições contendo 100 larvas cada uma, nas gerações dois e nove.

\begin{tabular}{|c|c|c|c|c|c|c|c|c|c|}
\hline \multirow{2}{*}{ Dieta } & \multirow{2}{*}{ Substrato } & \multicolumn{2}{|c|}{ Ciclo de vida (dias) } & \multicolumn{2}{|c|}{ Emergência (\%) } & \multicolumn{2}{|c|}{ Número de 6 vulos/femea } & \multicolumn{2}{|c|}{ Comprimento da asa (mm) } \\
\hline & & $F_{2}$ & $F_{9}$ & $\mathrm{~F}_{2}$ & F9 & F2 & F9 & $\mathbf{F}_{2}$ & F9 \\
\hline \multirow{4}{*}{ Terra } & Controle & $32,33 \pm 0,58 \mathrm{a}$ & $33,33 \pm 0,58 \mathrm{a}$ & $87,67 \mathrm{ab}$ & $75,67 \mathrm{a}$ & $195,00 \pm 10,40 \mathrm{a}$ & $174,40 \pm 28,22 a$ & $2,79 \pm 0,03 \mathrm{ab}$ & $2,70 \pm 0,12 \mathrm{a}$ \\
\hline & Mistura & $33,00 \pm 0,00 \mathrm{ab}$ & $34,00 \pm 0,00 \mathrm{ab}$ & $86,67 \mathrm{a}$ & $72,67 \mathrm{a}$ & $184,90 \pm 14,67 \mathrm{a}$ & $205,60 \pm 13,86 \mathrm{~b}$ & $2,77 \pm 0,06 \mathrm{ac}$ & $2,82 \pm 0,08 \mathrm{a}$ \\
\hline & Mucuna & $34,33 \pm 0,58 \mathrm{ab}$ & $34,67 \pm 0,58 \mathrm{ab}$ & $71,67 \mathrm{ab}$ & $70,00 \mathrm{a}$ & $104,40 \pm 13,29 b$ & $181,90 \pm 33,15 a b$ & $2,40 \pm 0,04 d$ & $2,70 \pm 0,09 \mathrm{a}$ \\
\hline & Batata & $37,33 \pm 0,58 b$ & $35,33 \pm 0,58 \mathrm{~b}$ & $60,00 \mathrm{~b}$ & $70,33 a$ & $184,80 \pm 10,66 \mathrm{a}$ & $197,80 \pm 9,24 \mathrm{ab}$ & $2,67 \pm 0,05 \mathrm{~cd}$ & $2,83 \pm 0,06 \mathrm{~b}$ \\
\hline \multirow{4}{*}{ Areia A } & Controle & $32,67 \pm 0,58 \mathrm{ab}$ & $31,00 \pm 0,00 \mathrm{a}$ & $85,00 \mathrm{ab}$ & $84,33 a$ & $179,70 \pm 13,17 \mathrm{a}$ & $159,70 \pm 28,74 a b$ & $2,78 \pm 0,03 \mathrm{a}$ & $2,72 \pm 0,05 a b$ \\
\hline & Mistura & $32,00 \pm 0,00 \mathrm{a}$ & $33,00 \pm 0,00 \mathrm{ab}$ & $84,33 a$ & $76,00 \mathrm{ab}$ & $187,00 \pm 19,98 \mathrm{a}$ & $189,40 \pm 17,42 \mathrm{a}$ & $2,80 \pm 0,03 \mathrm{a}$ & $2,77 \pm 0,05 \mathrm{a}$ \\
\hline & Mucuna & $36,67 \pm 1,15 \mathrm{ab}$ & $35,33 \pm 0,58 \mathrm{ab}$ & $76,00 \mathrm{ab}$ & $64,00 \mathrm{ab}$ & $99,44 \pm 14,69 b$ & $183,10 \pm 37,48 \mathrm{ab}$ & $2,41 \pm 0,04 b$ & $2,70+0,17 \mathrm{ab}$ \\
\hline & Batata & $37,67 \pm 1,15 b$ & $36,33 \pm 0,58 b$ & $69,67 \mathrm{~b}$ & $63,00 \mathrm{~b}$ & $113,90 \pm 14,80 \mathrm{~b}$ & $158,50 \pm 14,55 b$ & $2,36 \pm 0,07 b$ & $2,64 \pm 0,07 \mathrm{~b}$ \\
\hline \multirow{4}{*}{ Areia B } & Controle & $32,33 \pm 0,58 \mathrm{ab}$ & $31,33 \pm 0,58 a$ & $79,00 \mathrm{ab}$ & $83,00 \mathrm{a}$ & $187,40 \pm 12,39 \mathrm{a}$ & $144,60 \pm 14,00 a$ & $2,75 \pm 0,03 a$ & $2,55 \pm 0,07 a$ \\
\hline & Mistura & $32,00+0,00 \mathrm{ab}$ & $33,00+0,00 \mathrm{ab}$ & $85,33 a$ & $85,33 \mathrm{a}$ & $196,70 \pm 13,37 c$ & $211,40 \pm 8,77 \mathrm{~b}$ & $2,78 \pm 0,05 \mathrm{a}$ & $2,85 \pm 0,05 b$ \\
\hline & Mucuna & $30,00+1,00 \mathrm{a}$ & $34,00 \pm 0,00 \mathrm{ab}$ & $76,33 \mathrm{ab}$ & $70,00 \mathrm{ab}$ & $170,50 \pm 15,71 \mathrm{ab}$ & $206,80 \pm 12,87 \mathrm{~b}$ & $2,65 \pm 0,08 \mathrm{~b}$ & $2,79 \pm 0,07 \mathrm{bc}$ \\
\hline & Batata & $37,33 \pm 0,58 b$ & $37,67 \pm 0,58 b$ & $61,33 \mathrm{~b}$ & $65,00 \mathrm{~b}$ & $157,60 \pm 15,38 \mathrm{~b}$ & $163,60 \pm 16,77 \mathrm{a}$ & $2,64 \pm 0,06 \mathrm{~b}$ & $2,64 \pm 0,06 \mathrm{ac}$ \\
\hline
\end{tabular}

* Dentro de cada substrato, médias seguidas da mesma letra, em coluna, não diferem entre si (Kruskal Wallis, comparação múltipla à $5 \%$ ).

Para o parâmetro porcentagem de emergência houve diferenças significativas em cinco casos, sendo que, em quatro deles a porcentagem de emergência foi maior na dieta mistura em relação a dieta batata; no outro a porcentagem de emergência das moscas da dieta controle foi maior que as da dieta batata.

Já para os parâmetros número de óvulos e tamanho do adulto, houve diferenças significativas entre as dietas em seis casos. Os resultados foram menos uniformes nos dois parâmetros, porém, na maioria deles a dieta mistura foi superior a pelo menos uma das outras.

Avaliando-se, de maneira conjunta, os resultados de todos os parâ- 
metros, verifica-se que as melhores dietas para as larvas desta espécie foram a controle e a mistura, e a pior delas foi a batata. A dieta mucuna apresentou de modo geral, resultados intermediários para os parâmetros estudados.

É difícil determinar a causa do sucesso das dietas controle e mistura em relação as demais, devido às características particulares de cada uma e também, pelas características de sua preparação. Porém é provável que pelo fato dessas ramas terem sido lavadas e autoclavadas, quando foram oferecidas isoladamente, elas não reuniram condições apropriadas para uma boa fermentação, o que deve ter ocorrido quando oferecidas em conjunto (dietas mistura e controle). Além disso, quando oferecidas em conjunto, elas podem apresentar uma complementação de nutrientes, de modo a tornar a qualidade destas dietas superior as outras.

Um dos motivos para justificar a melhor fermentação das dietas mistura e controle poderia ser o fato das ramas em conjunto terem uma relação carbono/nitrogênio mais adequada do que as dietas mucuna e batata. Em Bradysia confinis foram feitos estudos que mostram a preferência desta espécie pelo consumo de folhas pouco decompostas, além desta preferência estar associada com uma relação ótima de $\mathrm{C} / \mathrm{N}$ existente nestas folhas em decomposição (DELEPORTE, 1987). Deste modo, pode-se sugerir que a relação C/N requerida por $B$. hygida seja semelhante a de $B$. confinis. Ainda, outra justificativa para o sucesso das dietas mistura e controle poderia ser o ambiente mais favorável ao desenvolvimento de certos microrganismos apesar de, na alimentação natural de $B$. confinis não ter sido verificada a necessidade deles (DELEPORTE, 1988). Com relação ao requerimento de microorganismos pela B. hygida, algumas observações feitas no laboratório indicam que eles são necessários (ANDRADE, com. pes.).

O fato da dieta batata ter proporcionado a pior performance está, de certo modo, contrariando os resultados da parte I do trabalho, onde ela teve o mesmo desempenhọ que as outras em, pelo menos, três parâmetros. Porém, os novos resultados demonstraram que quando testada isoladamente (sem a presença de terra) seu desempenho é o pior das quatro dietas. Isto confirma as observações empíricas que atribuiam à rama de batata, uma das causas dos problemas de manutenção da criação de $B$. hygida.

A comparação da dieta controle com a dieta mistura, ambas adequadas para a criação de $B$. hygida, mostra que tanto a terra como os aditivos utilizados na dieta controle são dispensáveis, uma vez que uma dieta constituída apenas das duas ramas lavadas e autoclavadas obteve igual sucesso na criação desta espécie.

Com relação a criação de Sciaridae, há muito tempo, tentativas de se obter criações estáveis tem sido feitas. Por exemplo: mistura de esterco com palha, inoculada com micélio de cogumelo para criar Bradysia coprophila (THOMAS, 1929); folhas frescas ou fermentadas para criar Bradysia tritici (ELISOR, 1934); fezes de ovelha misturada com palha para Sciara sp. (BUTT, 1934); cogumelo moído, levedura e palha para a cultura de Bradysia coprophila 
e Bradysia impatiens (SMITH-STOCKING, 1936; CARSON, 1946); rama de batata doce ou urtiga para Rhyncosciara angelae (LARA et al., 1965) e agar com farinha de milho junto com milho fermentado para criar espécies de Rhyncosciara (MATTINLY \& OGLE, 1969); rama de batata doce esterilizada e inoculada com cepas de levedo e de bactérias da própria rama para algumas espécies de Sciaridae (MORGANTE et al., 1970) e levedo de cerveja sobre um substrato de agar para criar Sciaridae hawaianos (STEFFAN, 1974). No entanto, não se tem informação de que qualquer desses métodos tenha mantido de maneira estável e contínua a criação desses Sciaridae, em laboratório.

Em todas estas dietas e também, nas aqui apresentadas, o que se tem de comum é o fato da utilização frequente de folhas fermentadas e leveduras ou fungos. Isto se explica pela própria biologia das espécies desta família, que vivem no solo, em vegetação decomposta.

Há, no entanto, controvérsias com relação aos hábitos alimentares das larvas desta família. De acordo com STEFFAN (1966) e EDWARDS (1974) as larvas de Sciaridae, geralmente, podem se alimentar de material vegetal em decomposição, excremento animal e fungos. WAAGE (1976) demonstrou o hábito coprófago dessas larvas registrando sua ocorrência em esterco fossilizado. LEATH \& NEWTON (1969) também demonstraram o hábito fitófago de algumas larvas desta família. O reconhecimento das espécies fitófagas é fácil. A maior dificuldade está em separar as espécies fitossaprófagas e coprófagas das micetófagas. Isto ocorre porque os fungos estão intimamente associados com o material vegetal decomposto e excremento animal, sendo que, este recurso poderia estar incluído nos dois últimos, dificultando o estabelecimento correto do hábito alimentar destas espécies (STEFFAN, 1974). Incluir a $B$. hygida em qualquer dessas categorias é muito prematuro, e pesquisas ainda devem ser realizadas para estabelecer os seus hábitos alimentares.

AGRADECIMENTOS. Agradecemos o apoio do Dr. Heni Sauaia, responsável pelo Laboratório de Biologia Celular, Departamento de Morfologia (FMRP-USP), onde este trabalho foi realizado; ao Dr. Gerson Mucillo, (FFCLRP-USP), pela orientação na parte estatística e ao $\mathrm{CNPq}$, pelo suporte financeiro (PIG V).

\section{REFERÊNCIAS BLIOGRÁFICAS}

AZEVEDO, E.M.V. \& J.R.P. PARRA. 1989. Influência da umidade em dois tipos de solo na emergência de Ceratitis capitata. Pesq. Agropec. Bras. 24 (3): 321-327.

BINNS, E.S. 1981. Fungus gnats (Diptera: Mycetophilidae/ Sciaridae) and the role of mycophagy in soil: a review. Rev. Ecol. Biol. Sol. 18 (1): 77-90.

BUTT, F.H. 1934. Embryology of Sciara (Sciaridae: Diptera). Ann. Entom. Soc. Amer. 27: 565-575.

CANTELO, W.W. 1988. Movement of Lycoriella mali (Diptera: Sciaridae) through mushroom-growing medium. J. Econ. Entom. 81 (1): 195-200. 
CARSON, H.L. 1946. The seletive elimination of inversion dicentric chromatids during meiosis in the eggs of Sciara impatiens. Genetics 31: 95-113.

DELEPORTE, S. 1987. Rôle du Diptère Sciaridae Bradysia confinis (Winn., Frey) dans la dégradation d'une litière de feuillus. Rev. Ecol. Biol. Sol. 24 (3): 341-358.

1988. Etude expérimentale de l'ajustement entre le cycle de Bradysia confinis (Diptera:Sciaridae) et l'evolution du substrat trophique (litière de feuillus): importance des microorganismes. Acta Oecol., Oecol. Gener., 9 (1):13-35.

DELEPORTE, S. \& C. ROULAND. 1991. Étude préliminaire de l'équipement digestif osidasique de Bradysia confinis (Diptera, Sciaridae): implications dans la dégradation de la matière organique. C.R. Acad. Sci. Paris, 312, Série III: $165-170$.

DENNIS, D.J. 1978. Observations of fungus gnat damage to glasshouse cucurbits. N.Z.J. exp. Agri. 6: 83-84.

EDWARDS, C.A. 1974. Macroarthropods. Biology of plant litter decomposition. London, Academic Press, vol. 2, p.533-554.

ELLISOR, L.O. 1934. Notes on the biology and control of Neosciara ocellaris (Comstock) (Diptera, Sciaridae). Iowa State Coll. Journ. Sci. 9: 25-36.

FRANCISCO, A.L. \& F.S. ZUCOLOTO. 1985. Obtenção de uma dieta artificial para Bradysia higida (Diptera:Sciaridae). Rev. Bras. Zool. 2 (6): 383-386.

GRAHAN, C.L. \& M.J. MCNEILL. 1972. Soybean crown and root damage by Bradysia coprophila. J.Econ. Entom. 65 (2): 597-599.

HOLLANDER, M. \& D.A. WOLFE. 1973. Nonparametric statistical methods. New York, John Wiley \& Sons, $1^{\text {a }}$ ed.

KEIL, C.B. \& M.H. OTHMAN. 1988. Effects of methoprene on Lycoriella mali (Diptera: Sciaridae). J.Econ. Entom. 81 (6): 1592-1597.

KENNEDY, M.K. 1974. Survival and development of Bradysia impatiens (Diptera: Sciaridae) on fungal and non-fungal food sources. Ann. Entom. Soc. Amer, 67 (5): 745-749.

LARA, F.S.J.; H. TAMAKI \& C. PAVAN. 1965. Laboratory culture of Rhynchosciara angelae. Amer. Natur. 99: 189-191.

LEATH, K.C. \& R.C. NEWTON. 1969. Interaction of a fungus gnat, Bradysia sp. (Sciaridae) with Fusarium spp. on alfafa and red cloner. Phytopatology 59: 257-258.

LUMMUS, P.F.; J.C.SMITH \& N.L.POWELL. 1983. Soil moisture and texture effects on survival of immature southern corn rootworms, Diabrotica undecimpunctata howardi Barber (Coleoptera: Chrysomelidae). Environ. Entom. 12: 1529-1531.

MATTINGLY, E. \& S.A. OGLE. 1969. A new culture method for Rhynchosciara. Ann. Entom. Soc. Amer. 62 (1): 94-96.

MORGANTE, J.S.; J. MARQUES; A.B. CUNHA \& I. ROMEO. 1970. Sobre a criação de alguns sciarideos (Diptera). Rev. Bras. Ent. 14 (4): 33-40.

NEUENSCHWANDER, P.; S. MICHELAKIS \& F. BIGLER. 1981. Abiotic 
factors affecting mortality of Dacus oleae larvae and pupae in the soil. Entom. Exper. Appl. 30: 1-9.

ROACH, S.H. \& R.B. CAMPBELL. 1983. Effects of soil compaction on bollworn (Lepidoptera: Noctuidae) moth emergence. Environ. Entom. 12: 1883-1886.

ROCK, G.C. 1972. Optimal proportion of dietary amino-acids. Insects and mite nutrition. Londres, North-Holand Publishing Company, p.183-197.

RUTHERFORD, T.A.; D.B. TROTTER \& J.M. WEBSTER. 1985. Monitoring fungus gnats (Diptera:Sciaridae) in cucumber greenhouses. Can. Entom. 117: $1387-1394$.

SAUAIA, H. \& M.A.R. ALVES. 1968. Description of a new species of Bradysia (Diptera: Sciaridae). Papéis Avulsos Zool, São Paulo 22: 85-88.

SIEGEL, S. 1975. Estatística não paramétrica (para ciências do comportamento). São Paulo, McGraw-Hill, 350p.

SMITH-STOCKING, H. 1936. Genetic studies on selective segregation of chromosomes in Sciara coprophila Lintner. Genetics 21: 421-443.

STEFFAN, W.A. 1966. A generic revision of the family Sciaridae (Diptera) of America North of Mexico. Univ. Calif. Publ. Entom. 44: 1-77.

. 1974. Studies and ecological notes on hawaiian Sciaridae (Diptera). Pacific Insects 16 (1): 41-50.

THOMAS, C.A. 1929. A method for rearing mushroom insects and mites. Entom. News 40: 222-225.

TRAMONTE, V.L.C.G. \& F.S. ZUCOLOTO. 1988. Estudos de carboidratos, como alimento para Bradysia hygida (Diptera: Sciaridae). Ciên. \& Cul. 40 (3): 273-275.

WAAGE, J.K. 1976. Insect remains from ground sloth dung. J. Paleontology 50 (5): 991-994.

WILKINSON, J.D. \& D.M. DAUGHERTY. 1970. The biology and imature stages of Bradysia impatiens (Diptera: Sciaridae). Ann. Entom. Soc. Amer. 63 (3): 656-660. 